\title{
Update of the Unitarity Triangle Analysis
}

\author{
A. J. Bevan, M. Bona \\ Queen Mary, University of London, E1 4NS, United Kingdom
}

M. Ciuchini

INFN, Sezione di Roma Tre, I-00146 Roma, Italy

D. Derkach, A. Stocchi

Laboratoire de l'Accélérateur Linéaire, IN2P3-CNRS et Université de Paris-Sud, BP 34, F-91898 Orsay Cedex, France

\section{E. Franco, L. Silvestrini}

INFN, Sezione di Roma, I-00185 Roma, Italy

\section{Lubicz, Cecilia Tarantino*}

Dipartimento di Fisica, Università Roma Tre, and INFN, I-00146 Roma, Italy

\section{G. Martinelli}

Dipartimento di Fisica, Università di Roma “La Sapienza”, and INFN, I-00185 Roma, Italy

\section{F. Parodi, C. Schiavi}

Dipartimento di Fisica, Università di Genova and INFN, I-16146 Genova, Italy

\section{Pierini}

CERN, CH-1211 Geneva 23, Switzerland

\section{Sordini}

IPNL-IN2P3 Lyon, France

\section{Vagnoni}

INFN, Sezione di Bologna, I-40126 Bologna, Italy

We present the status of the Unitarity Triangle Analysis (UTA), within the Standard Model (SM) and beyond, with experimental and theoretical inputs updated for the ICHEP 2010 conference . Within the SM, we find that the general consistency among all the constraints leaves space only to some tension (between the UTA prediction and the experimental measurement) in $\operatorname{BR}(B \rightarrow \tau v)$, $\sin 2 \beta$ and $\varepsilon_{K}$. In the UTA beyond the SM, we allow for New Physics (NP) effects in $\Delta F=2$ processes. The hint of NP at the $2.9 \sigma$ level in the $B_{S}-\bar{B}_{S}$ mixing turns out to be confirmed by the present update, which includes the new D0 result on the dimuon charge asymmetry but not the new CDF measurement of $\phi_{s}$, being the likelihood not yet released.

35th International Conference of High Energy Physics - ICHEP2010,

July 22-28, 2010

Paris France 


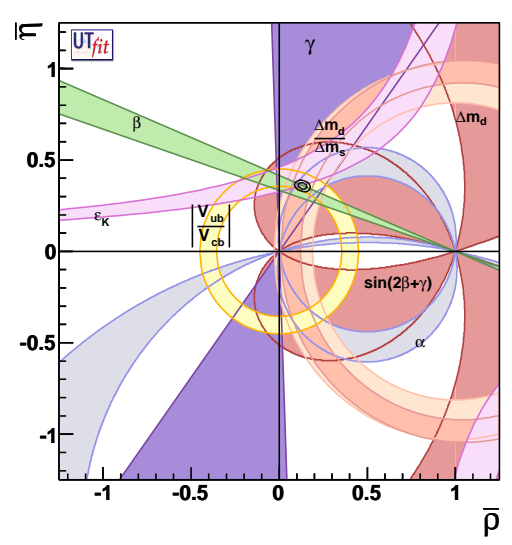

Figure 1: Results of the UTA within the SM. The contours display the selected $68 \%$ and $95 \%$ probability regions in the $(\bar{\rho}, \bar{\eta})$-plane. The $95 \%$ probability regions selected by the single constraints are also shown.

We present an update of the Unitarity Triangle Analysis (UTA) performed by the UTfit collaboration following the method described in refs. [1, 2]. Within the Standard Model (SM), we have included in $\varepsilon_{K}$ the contributions of $\xi$ and $\phi_{\varepsilon} \neq \pi / 4$ which, as pointed out in [3], decrease the SM prediction for $\varepsilon_{K}$ by $\sim 8 \%$. We have also included the long-distance contribution calculated more recently in [4], which softens the $8 \%$ reduction to $6 \%$. In a new paper [5] the perturbative calculation of the NNLO QCD corrections to the box diagram involving a top and a charm quark has been computed. This contribution, which is found to increase the theoretical prediction of $\varepsilon_{K}$ by $3 \%$, is not yet included in the UTA.

We observe, as main result of the UTA, that the CKM matrix turns out to be consistently overconstraint and the CKM parameters $\bar{\rho}$ and $\bar{\eta}$ are accurately determined: $\bar{\rho}=0.132 \pm 0.020$, $\bar{\eta}=0.358 \pm 0.012$ [6]. The UTA has thus established that the CKM matrix is the dominant source of flavour mixing and CP-violation and that New Physics (NP) effects can at most represent a small correction to this picture. We note, however, that the new contributions in $\varepsilon_{K}$ generate some tension in particular between the constraints provided by the experimental measurements of $\varepsilon_{K}$ and $\sin 2 \beta$ (see fig. 1). As a consequence, the indirect determination of $\sin 2 \beta$ turns out to be larger than the experimental value by $\sim 2.6 \sigma$. We observe that the updated lattice average of the bag-parameter $B_{K}$ [7] further enhances this $\varepsilon_{K}-\sin 2 \beta$ tension. This is due to the fact that new unquenched results, though compatible with older quenched results, tend to lie below them.

Recently, we have shown [8] how to use the UTA to improve the prediction of $\operatorname{BR}(B \rightarrow \tau \nu)$ in the SM, thanks to a better determination of $\left|V_{u b}\right|$ and $f_{B}$. Within the SM the UTA prediction for $\operatorname{BR}(B \rightarrow \tau \nu)$ is found to deviate from the experimental measurement [9] by $\sim 3.2 \sigma$. Even allowing for minimal flavour violating NP effects, a $\sim 3.0 \sigma$ deviation from the experimental value is found. Moreover, it is interesting to note that a large value of $\left|V_{u b}\right|$ (which is closer to some inclusive determinations) would reduce this deviation but it would enhance the tension in $\sin 2 \beta$.

We now present the update of the NP UTA, that is the UTA generalized to include possible NP effects. This analysis consists first in generalizing the relations among the experimental observ-

\footnotetext{
* Speaker.
} 
ables and the elements of the CKM matrix, introducing effective model-independent parameters that quantify the deviation of the experimental results from the SM expectations. The possible NP effects considered in the analysis are those entering neutral meson mixing. Thanks to recent experimental developments, in fact, these $\Delta F=2$ processes turn out to provide stringent constraints on possible NP contributions. In the case of $B_{d, s} \bar{B}_{d, s}$ mixing, a complex effective parameter is introduced, defined as

$$
C_{B_{d, s}} e^{2 i \phi_{B_{d, s}}}=\frac{\left\langle B_{d, s}\left|H_{e f f}^{f u l l}\right| \bar{B}_{d, s}\right\rangle}{\left\langle B_{d, s}\left|H_{e f f}^{S M}\right| \bar{B}_{d, s}\right\rangle},
$$

being $H_{e f f}^{S M}$ the SM $\Delta F=2$ effective Hamiltonian and $H_{\text {eff }}^{\text {full }}$ its extension in a general NP model, and with $C_{B_{d, s}}=1$ and $\phi_{B_{d, s}}=0$ within the SM. All the mixing observables are then expressed as a function of these parameters and the SM ones (see refs. $[10,11,12]$ for details). In a similar way, for the $K-\bar{K}$ system, one can write

$$
C_{\varepsilon_{K}}=\frac{\operatorname{Im}\left[\left\langle K\left|H_{e f f}^{f u l l}\right| \bar{K}\right\rangle\right]}{\operatorname{Im}\left[\left\langle K\left|H_{e f f}^{S M}\right| \bar{K}\right\rangle\right]}, \quad C_{\Delta m_{K}}=\frac{\operatorname{Re}\left[\left\langle K\left|H_{\text {eff }}^{\text {full }}\right| \bar{K}\right\rangle\right]}{\operatorname{Re}\left[\left\langle K\left|H_{\text {eff }}^{S M}\right| \bar{K}\right\rangle\right]},
$$

with $C_{\varepsilon_{K}}=C_{\Delta m_{K}}=1$ within the SM.

In this way, the combined fit of all the experimental observables selects a region of the $(\bar{\rho}, \bar{\eta})$ plane $(\bar{\rho}=0.135 \pm 0.040, \bar{\eta}=0.374 \pm 0.026)$ which is consistent with the results of the SM analysis, and it also constraints the effective NP parameters.

For $K-\bar{K}$ mixing, the NP parameters are found in agreement with the SM expectations. In the $B_{d}$ system, the mixing phase $\phi_{B_{d}}$ is found $\simeq 1.8 \sigma$ away from the SM expectation, reflecting the tension in $\sin 2 \beta$ discussed above.

The $B_{s}$-meson sector, where the tiny SM mixing phase $\sin 2 \beta_{s} \simeq 0.041(4)$ could be highly sensitive to a NP contribution, represents a privileged environment to search for NP. In this sector, an important experimental progress has been achieved at the Tevatron collider since 2008 when both the CDF [13] and D0 [14] collaborations published the two-dimensional likelihood ratio for the width difference $\Delta \Gamma_{s}$ and the phase $\phi_{s}=2\left(\beta_{s}-\phi_{B_{s}}\right)$, from the tagged time-dependent angular analysis of the decay $B_{s} \rightarrow J_{\psi} \phi$.

In 2009 the update of the UTfit analysis of ref. [15], combining the CDF and D0 results including the D0 two-dimensional likelihood without assumptions on the strong phases, yielded $\phi_{B_{s}}=(-69 \pm 7)^{\circ} \cup(-19 \pm 8)^{\circ}$, which is $2.9 \sigma$ away from the SM expectation $\phi_{B_{s}}=0$.

In 2010 two surprising news have arrived from the CDF and D0 experiments. On the one hand the new CDF measurement [16] based on an enlarged $\left(5.2 \mathrm{fb}^{-1}\right)$ data sample has provided a reduced significance of the deviation, from $1.8 \sigma$ to $\simeq 1 \sigma$. On the other hand D0 has performed a new measurement of the dimuon charge asymmetry $a_{\mu \mu}$ [17], which points to a large value of $\phi_{B_{s}}$, but also to a value for the width difference $\Delta \Gamma_{s}$ that is significantly larger than the SM prediction. If confirmed, the latter result would lead to one of the two (unlikely) explanations: either a huge NP contribution shows up in the tree-level observable $\Delta \Gamma_{s}$ or the operator product expansion badly fails (while it turns out to work well in describing b-hadron lifetimes where the same theoretical approach is adopted for the diagonal matrix element $\Gamma_{11}$ instead of $\Gamma_{12}$ ).

It will be interesting to see if these CDF and D0 results will be confirmed once the Tevatron measurements will improve. At the moment the new CDF result is not included in the analysis 


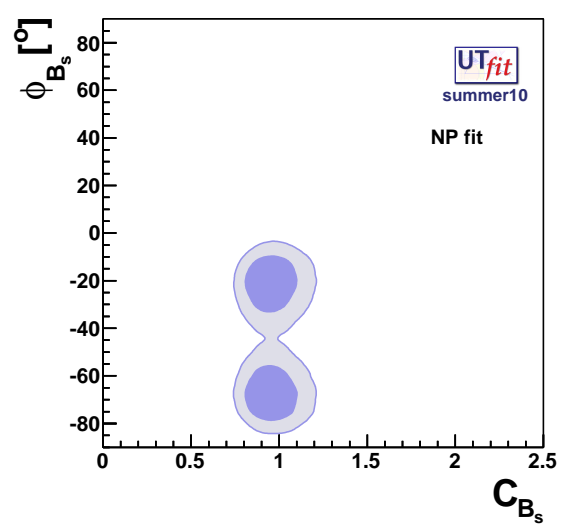

Figure 2: $68 \%$ (dark) and 95\% (light) probability regions in the $\left(C_{B_{S}}, \phi_{B_{s}}\right)$-plane.

since the $\Delta \Gamma_{s}-\phi_{s}$ likelihood has not been released yet. Including the D0 result for $a_{\mu \mu}$, we find $\phi_{B_{s}}=(-68 \pm 8)^{\circ} \cup(-20 \pm 8)^{\circ}$, which confirms the $2.9 \sigma$ deviation from the SM expectation $\phi_{B_{s}}=0$ (see fig. 2).

\section{References}

[1] M. Ciuchini et al., JHEP 0107 (2001) 013 [hep-ph/0012308].

[2] M. Bona et al. [UTfit Collaboration], JHEP 0507 (2005) 028 [hep-ph/0501199].

[3] A. J. Buras and D. Guadagnoli, Phys. Rev. D 78 (2008) 033005 [0805.3887 [hep-ph]].

[4] A. J. Buras, D. Guadagnoli and G. Isidori, Phys. Lett. B 688 (2010) 309 [arXiv:1002.3612 [hep-ph]].

[5] J. Brod and M. Gorbahn, arXiv:1007.0684 [hep-ph].

[6] The UTfit Collaboration, http://www.utfit.org/.

[7] V. Lubicz, PoS LAT2009 (2009) 013 [arXiv:1004.3473 [hep-lat]].

[8] M. Bona et al. [UTfit Collaboration], Phys. Lett. B 687 (2010) 61 [arXiv:0908.3470 [hep-ph]].

[9] The Heavy Flavour Averaging Group (HFAG), http://www.slac.stanford.edu/xorg/hfag/. G. De Nardo on behalf of the BaBar Collaboration, these proceedings.

[10] M. Bona et al. [UTfit Collaboration], JHEP 0603 (2006) 080 [hep-ph/0509219].

[11] M. Bona et al. [UTfit Collaboration], Phys. Rev. Lett. 97 (2006) 151803 [hep-ph/0605213].

[12] M. Bona et al. [UTfit Collaboration], JHEP 0803 (2008) 049 [0707.0636 [hep-ph]].

[13] T. Aaltonen et al. [CDF Collaboration], Phys. Rev. Lett. 100 (2008) 161802 [0712.2397 [hep-ex]].

[14] V. M. Abazov et al. [D0 Collaboration], Phys. Rev. Lett. 101 (2008) 241801 [0802.2255 [hep-ex]].

[15] M. Bona et al. [UTfit Collaboration], PMC Phys. A 3 (2009) 6 [arXiv:0803.0659 [hep-ph]].

[16] G. Giurgiu on behalf of the CDF Collaboration, these proceedings.

[17] V. M. Abazov et al. [D0 Collaboration], Phys. Rev. Lett. 105 (2010) 081801 [arXiv:1007.0395 [hep-ex]]. 